\title{
de agua
}
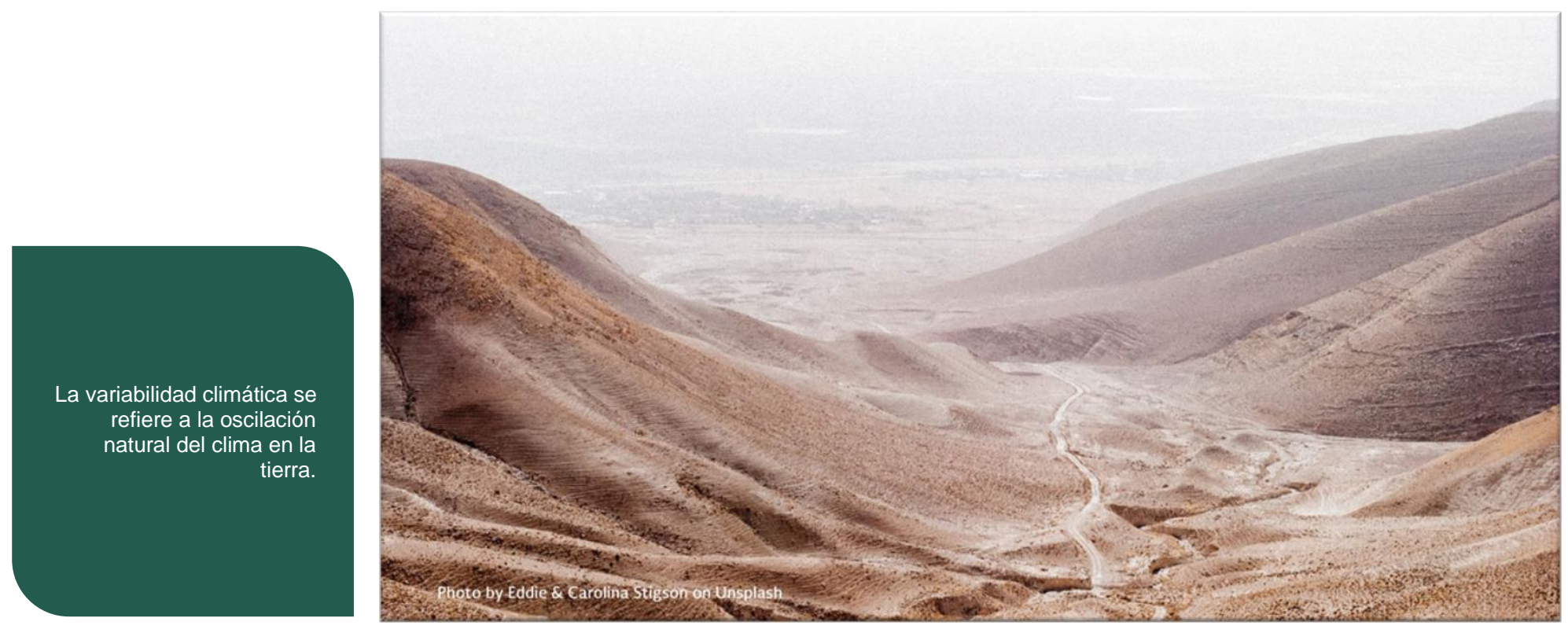

Sin importar el tipo de clima bajo el que nos encontremos en el planeta, árido o húmedo, estudios publicados recientemente a partir de información proveniente de satélites y estaciones de monitoreo en tierra han detectado un declive importante en el almacenamiento terrestre de agua (superficial y subterráneo), el cual es más grave en zonas áridas.

Estas zonas cubren más del 40 \% de la superficie terrestre y, tal como lo demostró Schimel (2010), desempeñan un papel preponderante en el secuestro de carbono a escala global. Por otro lado, estas zonas tienen un gran estrés hídrico (Huang et al., 2016) y presentan una alta vulnerabilidad ambiental y económica, al mismo tiempo que son el hogar de un tercio de la población del planeta (UNEP, 2011). Famiglietti (2004) dio cuenta de que en estos climas se presentan la mayor parte de los puntos críticos de declive en el almacenamiento de agua, utilizando para ello datos de la misión satelital GRACE de la Agencia Espacial Norteamericana (NASA, por sus siglas en inglés). Este declive en la cantidad de agua en ríos y lagos, glaciares, suelo, bosques y acuíferos tiene efectos no solo en la salud de los ecosistemas sino en la estabilidad socioeconómica de las localidades que sufren este fenómeno (Cosgrove y Loucks, 2015, Gleeson et al., 2012).

Existe un amplio espectro de procesos que pueden perturbar el ciclo hidrológico y el balance hídrico a nivel cuenca o acuífero, que dan como resultado un declive en el almacenamiento de agua. Entre ellos están la disminución del volumen de agua asegurada en los glaciares (Hugonnet et al., 2021), la sobreexplotación de acuíferos (Wada et al., 2012) la disminución de lagos (Wada et al., 2017) y las sequías 
meteorológicas (Rodell et al., 2018). Sin embargo, es importante destacar que estos procesos son manifestaciones claras de tres forzamientos principales: la variabilidad climática, el calentamiento global y las actividades humanas (Chew y Small, 2014; Khandu et al., 2016; Rodell et al., 2018).

La variabilidad climática se refiere a la oscilación natural del clima en la tierra (p. ej. la oscilación de El Niño, que afecta el almacenamiento de agua a través de la teleconección con las variables climáticas, en particular la precipitación). Los efectos de estas variaciones suelen ser transitorias, pero con efectos que alteran la tendencia en el almacenamiento durante años o décadas (Reager et al., 2016). En contraste, los impactos del calentamiento global y las actividades humanas son más seculares, en el primer caso, como lo indica el retroceso de los glaciares en el último siglo y, en el segundo caso, por el incremento continuo en la demanda de agua que resulta del crecimiento poblacional y que agrava la escasez hídrica en zonas áridas y semiáridas del planeta (Wada et al., 2016).

Uno de los mayores retos en la hidrología consiste en la disección clara de la proporción de cada uno de estos forzamientos al declive observado en el almacenamiento de agua, en virtud de que es altamente probable que los tres estén entrelazados y su importancia relativa varíe entre regiones y países. Desagregar las contribuciones de cada uno de los forzamientos es crítico para todas las naciones, pues permite evaluar las compensaciones entre la conservación ambiental de un territorio y su desarrollo social.

Investigaciones recientes confirman que, en el caso de zonas húmedas, el calentamiento global representa el principal forzamiento en el declive de almacenamiento de agua, y resulta de los cambios en la masa de los glaciares que están en estas regiones y que cubren $4 \%$ de su área total (aproximadamente $210,000 \mathrm{~km}^{2}$ ). Para estas regiones húmedas, tan solo en el periodo de tiempo definido entre los años 2002 y 2016 , se registró un incremento en la temperatura de la tierra de $0.6{ }^{\circ} \mathrm{C}$, lo que dio lugar a una pérdida en la masa de los glaciares de aproximadamente $136.4 \pm 23.8 \mathrm{Gt} / \mathrm{año}$. A pesar de una limitada cobertura espacial de los glaciares en estas zonas (4\% del área), esta pérdida de almacenamiento de agua en los glaciares coincide con el declive observado por los satélites en el almacenamiento terrestre. En el 96 \% del área restante de estas zonas húmedas, que no cuentan con glaciares, los forzamientos de la variabilidad climática y los impactos antropogénicos parecen contrarrestarse entre ellos, dando lugar a un equilibrio neto en el almacenamiento de agua (superficial y subterráneo).

Por otro lado, en las zonas áridas, el forzamiento predominante en la pérdida de almacenamiento de agua está dado por las actividades humanas. En esos mismos quince años de análisis (2002-2016) se encontró que en regiones sin presencia de glaciares las actividades antropogénicas, entre las que destacan la extracción inmoderada de agua de cuencas y acuíferos, así como la mala gestión de embalses, detonaron una pérdida de $104.3 \pm 5.3 \mathrm{Gt} / \mathrm{año}$, de los cuales el $90 \%$ se originaron en las zonas áridas (94.3 $\pm 3.0 \mathrm{Gt} / \mathrm{año}$ ). Por si esto fuera poco, la mayor parte de esa pérdida en el almacenamiento terrestre de las zonas áridas se origina en zonas críticas, donde la tasa de consumo de agua excede los $10 \mathrm{~mm} / a n ̃ o$. De forma coincidente, en estas regiones del mundo se encuentran identificadas extracciones de agua no sustentables para satisfacer una demanda que se origina por diferentes actividades económicas, entre las que destacan el riego, la industria y el sector pecuario. Así, en las zonas áridas, una condición naturalmente complicada desde el punto de vista hídrico se amplifica por las necesidades hídricas para el buen funcionamiento de la sociedad y la economía, lo que da lugar al declive general en el almacenamiento de agua en estas regiones. 
De hecho, se ha identificado de forma global que de entre las actividades económicas que más inducen este declive en el almacenamiento de agua está el excesivo uso de agua para el riego agrícola. Comparado con otros usos de agua, el riego es generalmente el que demanda mayores consumos, y muchas de las extracciones son generadas para satisfacer las necesidades biofísicas de los cultivos, como la transpiración (Pokhrel et al., 2021). Lo anterior es particularmente cierto en las zonas áridas, en donde las demandas atmosféricas de agua son altas como resultado de la baja precipitación anual y la demanda de agua para riego. Está documentado que cerca del $88 \%$ de la sobreexplotación de acuíferos se presenta en zonas áridas del planeta, hecho que a su vez indica que, dentro de estas regiones, la pérdida inducida por actividades humanas (98.6 $\pm 0.9 \mathrm{Gt} /$ año) explica casi el $70 \%$ del declive de almacenamiento terrestre de agua ( $147.1 \pm 18.6 \mathrm{Gt} /$ año), valores consistentes publicados por Wada et al. (2010) y Wang et al. (2018). En zonas áridas, la impactante presencia de acuíferos sobreexplotados incide claramente sobre el declive del almacenamiento de agua, lo cual, a su vez, acentúa la severidad de las prácticas no sustentables de extracción, tal como está documentado para Irán, la India o Arabia Saudita. La aridez del clima da como resultado tasas de recarga de acuíferos extremadamente bajas (Döll et al., 2014). Por si esto fuera poco, los acuíferos en estas regiones tienden a tener el nivel freático a profundidades mayores, y solo pueden ser recargados como resultado de lluvias torrenciales (Bierkens y Wada, 2019).

En este sentido, podemos anticipar que, a lo largo de este siglo, conforme la población y el estándar de vida (que requiere agua) aumenten, el uso del agua para las actividades humanas se incrementará de forma exponencial (Wada and Bierkens, 2014). Esta situación nos impone la amplificación de una crisis que requiere la incorporación de un lente de sustentabilidad hídrica regional en todas las decisiones que detonen actividades económicas en territorio. Esto nos permitirá florecer y crear inversión en regiones con abundancia de agua y cuidarla en regiones dominadas por la escasez presente o futura.

Referencias

Bierkens, M. F. P., \& Wada, Y. (2019). Non-renewable groundwater use and groundwater depletion: A review. Environmental Research Letters, 14(6), 43. https://doi.org/10.1088/1748-9326/abla5f

Chew, C. C., \& Small, E. E. (2014). Terrestrial water storage response to the 2012 drought estimated from GPS vertical position anomalies. Geophysical Research Letters, 41(17), 6145-6151. https://doi.org/10.1002/2014gl061206

Cosgrove, W. J., \& Loucks, D. P. (2015). Water management: Current and future challenges and research directions. Water Resources Research, 51(6), 4823-4839. https://doi.org/10.1002/2014wr016869

Döll, P., Mueller Schmied, H., Schuh, C., Portmann, F. T., \& Eicker, A. (2014). Global-scale assessment of groundwater depletion and related groundwater abstractions: Combining hydrological modeling with information from well observations and GRACE satellites. Water Resources Research, 50(7), 5698-5720. https://doi.org/10.1002/2014wr015595

Famiglietti, J. S. (2004). Remote sensing of terrestrial water storage, soil moisture and surface waters. State of the Planet: Frontiers and Challenges in Geophysics, 150, 197-207. https://doi.org/10.1029/150gm16

Gleeson, T., Wada, Y., Bierkens, M. F., \& van Beek, L. P. (2012). Water balance of global aquifers revealed by groundwater footprint. Nature, 488(7410), 197-200. https://doi.org/10.1038/nature11295

Huang, J., Yu, H., Guan, X., Wang, G., \& Guo, R. (2016). Accelerated dryland expansion under climate change. Nature Climate Change, 6(2),166-171. https://doi.org/10.1038/nclimate2837

Hugonnet, R., McNabb, R., Berthier, E., Menounos, B., Nuth, C., Girod, L., et al. (2021). Accelerated global glacier mass loss in the early twenty-first century. Nature, 592(7856), 726-731. https://doi.org/10.1038/s41586-021-03436-z 
Khandu, Forootan, E., Schumacher, M., Awange, J. L., \& Muller Schmied, H. (2016). Exploring the influence of precipitation extremes and human water use on total water storage (TWS) changes in the GangesBrahmaputra-Meghna River Basin. Water Resources Research, 52(3),2240-2258. https://doi.org/10.1002/2015wr018113

Pokhrel, Y., Felfelani, F., Satoh, Y., Boulange, J., Burek, P., Gadeke, A., et al. (2021). Global terrestrial water storage and drought severity under climate change. Nature Climate Change, 11(3), 226-233. https://doi.org/10.1038/s41558-020-00972-w

Reager, J. T., Gardner, A. S., Famiglietti, J. S., Wiese, D. N., Eicker, A., \& Lo, M. H. (2016). A decade of sea level rise slowed by climate-driven hydrology. Science, 351(6274), 699-703. https://doi.org/10.1126/science.aad8386

Rodell, M., Famiglietti, J. S., Wiese, D. N., Reager, J. T., Beaudoing, H. K., Landerer, F. W., \& Lo, M. H. (2018). Emerging trends in global freshwater availability. Nature, 557(7707), 651-659. https://doi.org/10.1038/s41586-018-0123-1

Schimel, D. S. (2010). Drylands in the earth system. Science, 327(5964), 418-419. https://doi.org/10.1126/science.1184946

UNEP. (2011). Global drylands: A UN system-wide response.

Wada, Y., \& Bierkens, M. F. P. (2014). Sustainability of global water use: Past reconstruction and future projections. Environmental Research Letters, 9(10), 104003. https://doi.org/10.1088/17489326/9/10/104003

Wada, Y., Florke, M., Hanasaki, N., Eisner, S., Fischer, G., Tramberend, S., et al. (2016). Modeling global water use for the 21st century: The Water Futures and Solutions (WFaS) initiative and its approaches. Geoscientific Model Development, 9(1), 175-222. https://doi.org/10.5194/gmd-9-175-2016

Wada, Y., Reager, J. T., Chao, B. F., Wang, J., Lo, M.-H., Song, C., et al. (2017). Recent changes in land water storage and its contribution to sea level variations. Surveys in Geophysics, 38(1), 131-152. https://doi.org/10.1007/s10712-016-9399-6

Wada, Y., van Beek, L. P. H., \& Bierkens, M. F. P. (2012). Nonsustainable groundwater sustaining irrigation: A global assessment. Water Resources Research, 48(6), W00L06. https://doi.org/10.1029/2011wr010562 Wang, J., Song, C., Reager, J. T., Yao, F., Famiglietti, J. S., Sheng, Y., et al. (2018). Recent global decline in endorheic basin water storages. Nature Geoscience, 11(12), 926-932. https://doi.org/10.1038/s41561018-0265-7 